BNL --45154

DE91 001724

\title{
SCATTERING BY ANISOTROPIC GRAINS \\ IN BERYLLIUM MIRRORS
}

\author{
Eugene L. Church \\ U. S. Army, ARDEC, Dover, NJ 07801-5001 \\ Peter Z. Takacs \\ Brookhaven National Laboratory, Upton, NY 11973 \\ J. C. Stover
}

TMA Technologies, Inc., Bozeman, MT 59715

August 1990

*This research was supported by the U. S. Department of Energy: Contract No. DE-AC02-76CH00016. 


\section{DISCLAIMER}

This report was prepared as an account of work sponsored by an agency of the United States Government. Neither the United States Covernment nor any agency thereof, nor any of their employees, nor any of their contractors, subcontractors, or their employees, makes any warranty. express or implied, or assumes any legal liability or responsibility for the accuracy, completeness, or usefulness of any information, apparatus, product, or process disclosed, nr represents that its use would not infringe privately owned rights. Reference

Therein to any specific commercial product, process or service by trade name, tratefhark, manufacturer, or otherwise, does not necessarily constitute or imply its endorsement, recommendation, or favoring by the United States Government or any agency, contractor, or subcontractor thereof. The views and opinions of authors expressed herein do not necessarily state or reflect those of the United States Government or any agency, contractor or subcontractor thereof. 


\title{
SCATTERING BY ANISOTROPIC GRAINS IN BERYLLIUM MIRRORS*
}

\author{
E. L. Church \\ U. S. Army, ARDEC, Dover, NJ 07801-5001 \\ P. Z. Takacs \\ Brookhaven National Laboratory, Upton, NY 11973 \\ J. C. Stover \\ TMA Technologies, Inc., Bozeman, MT 59715
}

\begin{abstract}
Scattering from mirror surfaces arises from topographic and non-topographic sources. This paper considers the nontopographic scattering of beryllium mirrors modelled as a collection of randomly oriented bireflective grains. Simple scattering theory shows that this type of scattering scales as $\lambda^{-2}$, rather than as $\lambda^{-4}$ for topographic scattering, which means that it is relatively more important at long radiation wavelengths. Estimates of the intensity based an available short-wavelength values of the anisotropic optical constants of beryllium indicate that this type of scattering could dominate the topographic scattering from smooth surfaces at $\mathrm{CO}_{2}$ wavelengths.
\end{abstract}

\section{IN'RRODUCTION}

Beryllium mirrors appear to have unusual scattering properties.

In 1989; nirst at the RADC L arge Optics Conference [1] and later at the SPIE meeting in San Diego [2], Stover et al. reported that the BRDFs of $s$ :veral smooth beryllium mirrors showed more scattering at longer radiation wavelengths than expected using the wa'cength-scaling laws for topographic scatter. They called this "anomalous" scatter and speculated that it was due to subsurface damage.

At the same conferences, Church, Takacs and Leonard $[3,4]$ conipared direct scattering measurements with predictions based an optical profile measurements made at $6500 \mathrm{~A}$. They agreed for some mirrors, bui the beryllium mirror always scattered more than predicted. They attributed this to "non-topographic" scattering mechanisms; most likely the many small "pits" observed on the beryllium surface. These were a prime candidate since they were poorly sampled in the profile measurements and were so large that their scattering did not obey the weak-scattering scaling laws.

Chuirch and Takacs have also considered surface and volume inhomogeneities as sources of non-topographic scattering, and pointed ort 'hat these too can lead to scattering which falls off less rapidly with increasing radiation wavelength than topographic scattering [5,6]: Instead of scaiing with the familiar $\lambda^{-4}$ factor of topographic scatter, they can scale as $\lambda^{-2}$, which makes their contribution relatively more important at longer radiation wavelengths.

The present paper describes another non-iopographic scattering mechanisni which may lead to significant $\lambda^{-2}$ scattering from mirrors made of bireflective materials such as beryllium: scattering from the random orientation of optically anisotropic grains in the surface. Such grain structure is easily seen in beryllium surfaces using cross-polarized microscopy.

*This research was supported by the U. S. Department of Energy: Contract No. DE-AC02-76CH00016. 
In the past, the major factors limiting the performance of mirror surfaces have been their figure, finish, surface imperfections and contamination. As production methods improve, non-topographic contributions become relatively more important, especially at longer wavelengths. Understanding these mechanisms is an essential step in product improvement and guality control. Grain scattering, as discussed in this paper, is unique in that it arises not from isolated or external factors such as imperfections or contamination, but is intrinsic to the structure of the mirror material itself.

\section{SCALAR SCATTERING THEORY}

Simple scalar scattering theory leads to the following expression for the radiation reflected and scattered from a surface

$$
\frac{1}{I_{1}}\left\langle\frac{d I}{d \vec{\omega}}\right\rangle=\frac{\cos \theta}{\lambda^{2}}\left\langle\frac{1}{A}\left|\int_{A} d \vec{x} e^{12 x] \cdot x} e^{i \frac{\sin }{\lambda} \cos \theta \cdot z(x)} r(\vec{x})\right| 2\right|
$$

The first factor in the integrand is the diffraction kernel, the second is the phase modulation impressed on the reflected wavefront by the surface height fluctuations $Z$, and the third is the amplitude reflection coefficient of the surface, $r$. The spatial frequency $\vec{f}$ is related to the scattering geometry through the grating equations

$$
\left(\begin{array}{l}
f_{x} \\
f_{y}
\end{array}\right)=\frac{1}{\lambda}\left(\begin{array}{l}
\sin (\theta+\Delta \theta) \cdot \cos \Delta \varphi-\sin \theta \\
\sin (\theta+\Delta \theta) \cdot \sin \Delta \varphi
\end{array}\right)
$$

In the case of a perfect surface, $Z=0$ and $r=a$ constant which can be taken out of the integral. Equation 1 then reduces to a delta function which represents the specularly reflected beam. Scattering comes from the fact that $Z$ and $r$ depend on their position on the mirror surface which destroys the delicate interference required to generate only specular reflection.

In the perturbation limit the height and reflectivity factors are

$$
\begin{gathered}
e^{i \frac{4 \pi}{\lambda} \cos \theta \cdot z(\vec{r})}=1+i \frac{4 \pi}{\lambda} \cos \theta \cdot Z(\vec{x}) \\
r(\vec{x})=\langle r\rangle+[r(\vec{x})-\langle r\rangle]
\end{gathered}
$$

where $\langle r\rangle$ is the average reflectivity. Substituting these into Eq. 1 gives

$$
\frac{1}{I_{1}}\left\langle\frac{d I}{d \vec{\omega}}\right\rangle=\frac{\cos \theta}{\lambda^{2}} \delta(\vec{f})+\frac{16 \pi^{2}}{\lambda^{4}} \cos ^{3} \theta \cdot|<r>|^{2} \cdot S_{2}(\vec{f})+\frac{\cos \theta}{\lambda^{2}} \cdot S_{r}(\vec{f})
$$

where the first term is the specular term, the second is the scattering due to the topographic height fluctuations, and the third is that due to the spatial variations of the surface reflectivity [5-7].

The symbol $S$ stands for the power spectral density

$$
S_{Q}(\vec{f})=\lim _{A \rightarrow \infty}\left\langle\frac{1}{A}\left|\int_{A} d \vec{x} e^{12 \pi f \cdot x} Q(\vec{x})\right|^{2}\right\rangle
$$


The power spectrum of the height fluctuations is a well-known surface statistic: its shape is related to the spatial distribution of the roughness on the surface and its area is the mean-square roughness. Similarly, the spectrum of the reflectivity variations is related to the size and shape of the grains, and its area is the mean-square value of the refiectivity fluctuations.

The ratio of reflective to topographic scatter is

$$
\frac{(d I / d \vec{\omega})_{\text {rel }}}{(d I / d \vec{\omega})_{\text {ropo }}}=\frac{\left(\frac{\lambda}{4 \pi 00 \mathrm{c} \theta}\right)^{2}}{|\langle r\rangle|^{2}} \cdot \frac{S_{r}(\vec{f})}{S_{z}(\vec{f})}=\frac{\left\langle\left|\frac{r-\langle r\rangle}{\langle r\rangle}\right|^{2}\right\rangle}{\left(\left.4 \pi \frac{0}{\lambda} \cos \theta\right|^{2}\right.}
$$

where $\sigma$ is the root-mean-square (rms) surface roughness. This final form follows if we take the shape of the reflectivity spectrum to be the same as that of the topographic scatter. This is a reasonable approximation for the present feasibility argument and may not be too unrealistic considering that the height fluctuations of highly finished surfaces may be correlated with the grain structure responsible for reflective scatter.

Note the structure of the rinal result: The numerator is the mean square value of the reflectivity fluctuations relative to the average reflectivity, and the denominator is the expression for the topographic total-integrated-scatter (TIS).

\section{COMMENTS ON WAVELENGTH SCALING}

Equation 4 shows that topographic scattering involves a wavelength factor of $\lambda^{-4}$-the famous Rayleigh blue-sky factor - while reflective-scatter has a factor of only $\lambda^{-2}$. The importance of this difference in determining the relative importance of the two types of scattering has already been mentioned. But why is there a difference?

Comparison of Eqs. 1 and 3 shows that the total wavelength dependence comes from two sources: There is an overall factor of $\lambda^{-2}$ in Eq. 1 which comes from the two-dimensional nature of the scattering surface, and it is this factor which appears in reflective scatter. Topographic scatter, on the other hand, involves an additional factor of $\lambda^{-2}$ to account for the fact that the perturbation which gives rise to such scattering involves an additional length parameter-the surface height - which is compensated for by additional wavelength factors.

In the case of one-dimensional or corrugated surfaces the first factor becomes $\lambda^{-1}$, which is then the scaling for reflective scatter and leads to the well-known factor of $\lambda^{-3}$ for the topographic scattering from such surfaces. Other possibilities also exist $[5,6]$.

\section{ESTIMATION OF TIIE MEAN-SQUARE REFLECTIVITY FLUCTUATIONS}

When light is reflected from an anisotropic medium its refiectivity is governed by a matrix rather than an algebraic relationship:

$$
\left[\begin{array}{l}
E_{s} \\
E_{p}
\end{array}\right)_{r e f l}=\left(\begin{array}{l}
r_{s s} r_{s p} \\
r_{p s} r_{p p}
\end{array}\right)\left[\begin{array}{l}
E_{s} \\
E_{p}
\end{array}\right)_{\text {incid }}
$$

where the $r$ 's are amplitude reflection coefficients. For example, for $s$-polarized radiation in 


$$
\vec{E}_{r e f i}=\left[r_{s s} \hat{e}_{s}+r_{p s} \hat{e}_{p}\right] \cdot E_{s i n c l d}
$$

The first term on the right is s-polarized and the second is p-polarized. Together they describe the elliptical character of the reflected light. These $r$ factors are, in turn, functions of geometrical factors and the intrinsic ordinary, $r_{o}$, and extraordinary, $r_{e}$, reflection coefficients of the crystal grains.

The connection is quite complicated except in special cases [8]. For the present purposes we consider the case of normal incidence and where the optic axes of the grains lie in the surface plane, which lead to the simple expressions

$$
\begin{aligned}
& r_{s s}=r_{o} \cos ^{2} \psi+r_{e} \sin ^{2} \psi \\
& r_{p s}=\left(r_{o}-r_{e}\right) \sin \psi \cos \psi
\end{aligned}
$$

where $\psi$ is the angle between the optic axis and the plane of incidence defined by the $E$ vector of the incident radiation. It follows that the average values of these quantities are

$$
\begin{aligned}
& \left.<r_{s s}\right\rangle=\frac{1}{2}\left(r_{o}+r_{e}\right) \\
& \left.<r_{p s}\right\rangle=0
\end{aligned}
$$

and so

$$
\left\langle\left|r_{s s}-\left\langle r_{s s}\right\rangle\right|^{2}\right\rangle=\left\langle\left|r_{p s}-\left\langle r_{p s}\right\rangle\right|^{2}\right\rangle=\left.\frac{1}{8}\left|<r_{o}-r_{e}\right\rangle\right|^{2}
$$

Substituting these into Eq. 6 gives

$$
\frac{(d I / d \vec{\omega})_{\text {reft }}}{(d I / d \vec{\omega})_{\text {topo }}}=\frac{\frac{1}{2}\left|\frac{r_{0}-r_{e}}{r_{0}+r_{e}}\right|^{2}}{\left(4 \pi \frac{a}{2}\right)^{2}}
$$

The same result holds for $p$-polarized incident radiation at normal incidence.

\section{RESULTS FOR BERYLLIUM}

Figure 1 shows the magnitude of the numerator of Eq. 12 as a function of radiation wavelength for berylium, derived from data provided by Arakawa et al. [9], based in turn on the reflectivity measurements of Weaver et al. [10]. The dashed line indicates the value of $10^{-4}$ which is used below.

Figure 2 shows the division of roughness-wavelength space obtained from $\mathrm{Ec} .12$ using the solid and dashed data in Fig 1. For a given radiation wavelength the lines give the rms surface roughness which generates equal topographic and reflective scatter. Topographic scatter dominates for surfaces with roughness values lying above the lines, and reflective or grain scattering dominates for surfaces with roughnesses lying below the line.

There are two caveats here. First of all, the solid line is based on the direct measurements of the optical constants of beryllium which only cover low wavelengths, and second, it is based on the model that the optic axes of the grains all lie in the surface plane. Averaging that direction over the sphere would be expected to lower the solid line in Fig. 2. 
The dashed line corresponds to normalized rms reflectivity fluctuations having the value of $10^{-4}$ for all wavelengths. Although this particular value is arbitrary, the results in Fig. 2 show the small fluctuations in reflectivity which are required to dominate topographic scattcring at long radiation wavelengths - whatever their origin.

\section{FOLLOW.ON}

There is a crying need for measurements of the anisotropic optical constants of beryllium to confirm the earlier results of Weaver et al. and to extend them to longer wavelengths. Measurements of the wavelength dependence of the BRDFs of smooth flaw-free beryllium surfaces are essential for identifying various scattering mechanisms.

We are carrying through a vector scuttering calculation to determine polarization properties which may be useful for distinguishing different scattering mechanisms, as well as averaging over arbitrary distributions of the grain orientations. Extension to include the effects of impurities along the grain boundaries is also possible.

\section{ACKNOWLEDGEMENTS}

This work was supported in part by the USAF Rome Air Development Center (RADC) and the Optics MODIL at Oak Ridge National Laboratory and the U. S. Department of Energy under Contract No. DE-AC02-76CH00016.

\section{REFERENCES}

1. J. C. Stover, M. Bernt, D. McGary and J. Rifkin, "An Investigation of Anomalous Scatter from Beryllium Mirrors," presented at the RADC Large Optics Conference, July 1989, Proceedings RADC-TR-89-350, T-1-T-19, Dec. 1989.

2. J. C. Stover, M. Bernt, D. McGary and J. Rifkin, "An Investigation of Anomalous Scatter from Beryllium Mirrors," Scatter from Optical Components, John C. Stover, ed., Proc. SPIE 1165, 100-109 (1989).

3. E. L. Church, P. Z. Takacs and T. A. Leonard, "The Prediction of the BRDF from WYKO Profile Measurement," presented at the RADC Large Optics Conference, July 1989, Proceedings RADC-TR-89-350, S-1-S-23, Dec. 1989.

4. E. L. Church, P. Z. Takacs and T. A. Leonard, "The Prediction of BRDFs from Surface Profile Measurements," Scatter from Optical Components, John C. Stover, cd., Proc. SPIE 1165, 136-150 (1989).

5. E. L. Church and P. Z. Takacs, "Light Scattering from Surface and Subsurface Defects," presented at the ASPE Spring Conference on Subsurface Damage in Glass, Tucson, AZ, April 1989.

6. E. L. Church and P. Z. Takacs, "Light Scattering from Surface and Subsurface Defects," Scatter from Optical Components, John C. Stover., ed., Proc. SPIE 1165, 31-41 (1989).

7. References 5 and 6 point out that when the vertical dimension of the surface inhomogencities is much larger than the skin depth (columnular inhomogeneities), scattering can be described in terms of the variations of the surface reflectivity (reflective scattering). This is assumed to be the case in the present problem since the grain size of the mirrors examined is 10 's of $\mu \mathrm{m}$.

8. R. M. A. Azzam and N. M. Bashara, Ellipsometry and Polarized Light, (North Holland Publishing Company, Amsterdam, 1977), pp. 354 et seq. 
9. E. T. Arakawa, T. A. Callcott and Y. C. Chang, "The Optical Properties of Beryllium," Oak Ridge National Laboratory Report ORNL/OMIS-89/9. This report contains an error in the analysis of the Weaver data (ref. 10 below) which has been corrected in the present analysis.

10. J. A. Weaver, D. W. Lynch and R. Rosei, "Optical Properties of Single Crystal Be from 0.12 to 4.5 eV," Phys. Rev. B7, 3537-3540 (1973).

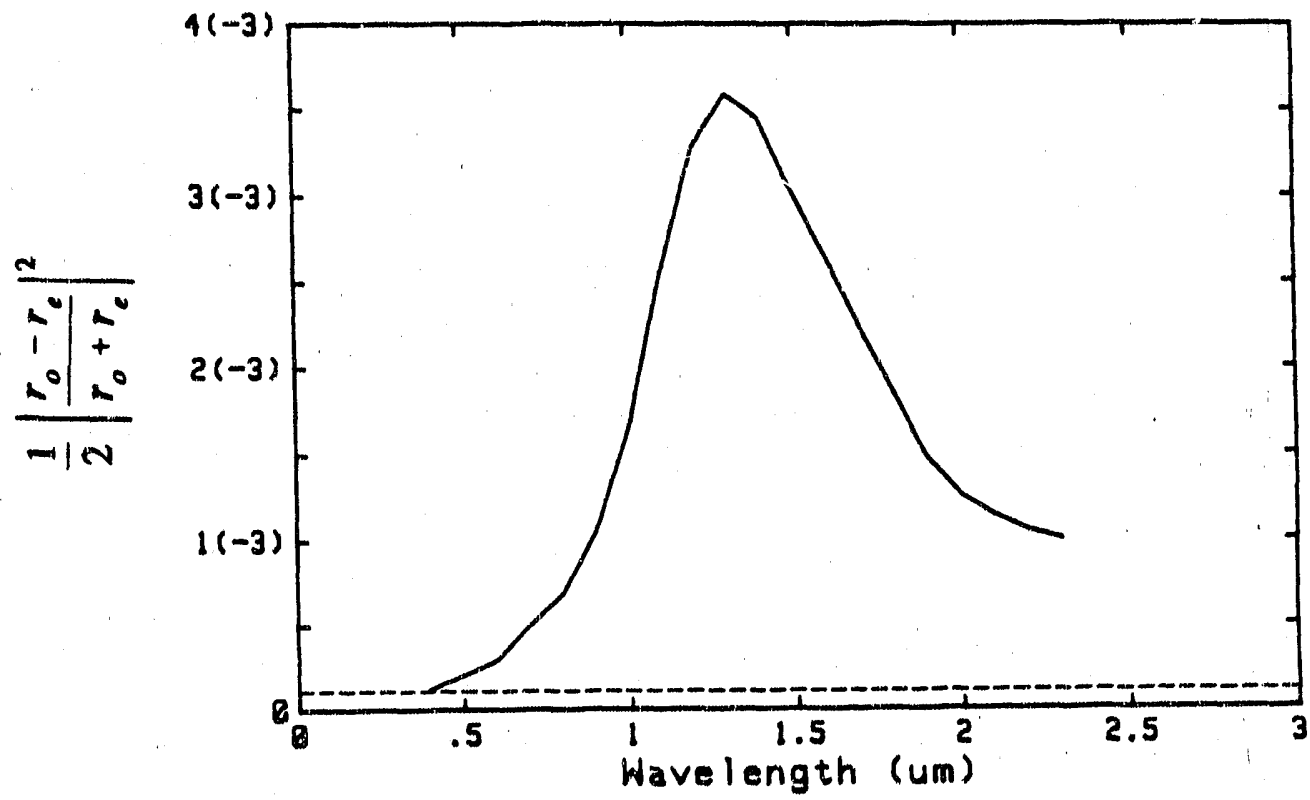

Figure 1. The mean-square value of the reflectivity fluctuations of beryllium as a function of radiation wavelength: Solid line derived from the data in Weaver [10]; dashed line indicates a constant value of $10^{-4}$.

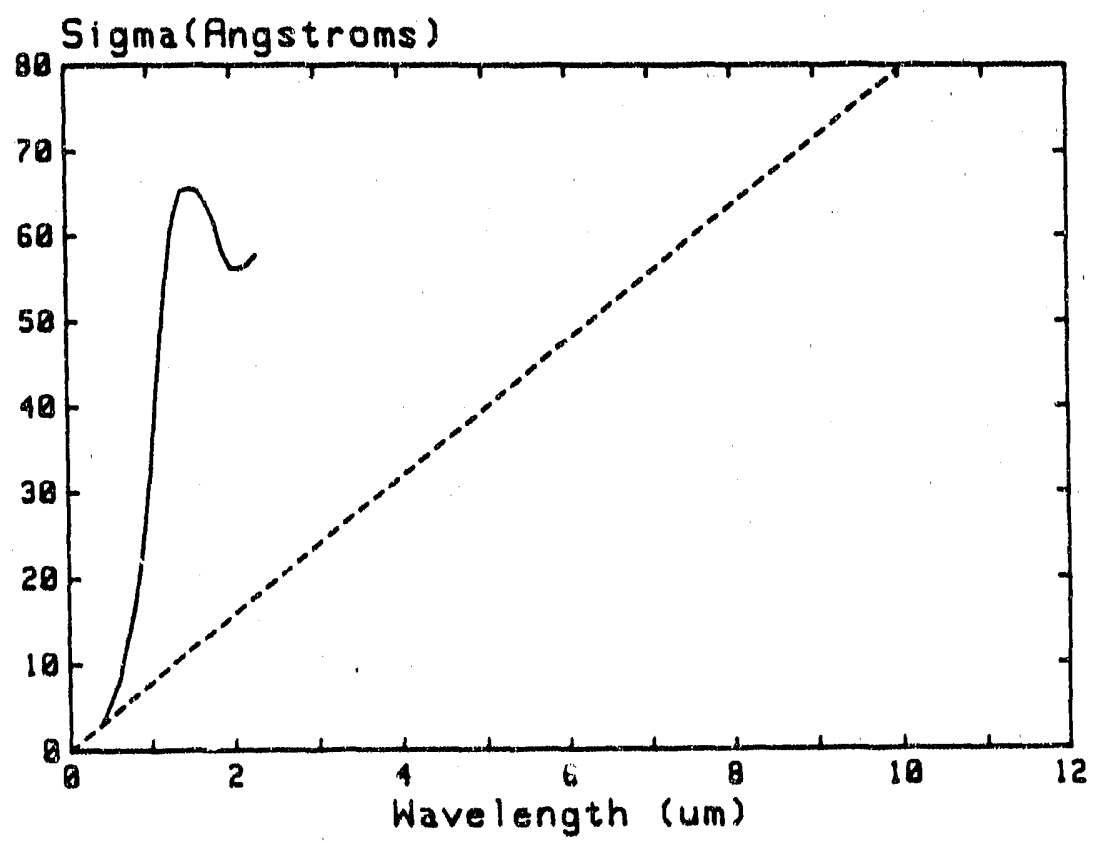

Figure 2. Root-mean-square roughness values which give equal topographic and reflective scattering. The solid and dashed lines correspond to the curves in Fig 1. 

